\title{
Quality Assurance and Institutional Research
}

\author{
Hester Geyser and Nicolene Murdoch
}

\section{Context and purpose}

Higher Education is facing ever-growing pressures concerning effectiveness, accountability and responsiveness to continuously changing national and institutional environments. Institutions are also faced with challenges related to budget allocation, staffing and improving quality delivery within constrained infrastructural environments. These challenges are supplemented by external factors such political unrest, which impact on the student experience. In these complex times, Institutional Research (IR) plays an integral role in the ways in which the university responds to these challenging times and makes decisions. Knight and Leimer (2010:110) consider IR as an important function that is essential to the accreditation process. It helps with decisions about programme offerings, and the support for teaching and learning. It can contribute to the institution's sound governance arrangements and raise its accountability standards. It can provide the information for conducting programme reviews and thus improve the quality of decisions made on the basis of evidence, leading, amongst other advantages, to sound initiatives for quality improvement. All these functions illustrate the close links between IR and quality assurance leading to quality assurance (QA) functions becoming integral to IR functions only in recent years.

Lange, Saavedra and Romano (2013:28-29) state that the implementation of various quality assurance activities heightened the need for adequate data to be captured by management information systems (MIS) and reported and interpreted by the IR practitioners at higher education institutions. Much of the data used in institutional and programme reviews are produced through a variety of IR functions. The challenged environment presents continuous risks to the students' experience at the institution and performance in the classroom. Accurate and informed decision making becomes increasingly important if the institution is to enable student performance and provide quality delivery.

In South Africa, the Higher Education Quality Committee (HEQC) introduced institutional audits in 2004. These audits constituted one of the mechanisms through which the national authority carried out its mandate for quality assurance. The audits focused on institutional capacity for quality management of its academic activities in relation to its vision, mission and goals. This served as an important impetus for the establishment of QA units at institutional level in many universities. Maturing processes necessitated collaboration between IR and QA to provide data on institutional effectiveness and efficiency. This collaboration and/or integration of QA and IR was further promoted by the need for IR data for external or national 
programme reviews by professional bodies. Lange et al. (2013:30), however, contend that it is often left to individual IR practitioners to provide data related to programmes with regard to student success, since evidence-based self-evaluation is a key element of quality reviews of institutions, programmes and modules. This is often because data are often not kept and archived consistently or because the IR practitioners are some of the few who possess the expertise to extract this data. They have access to information and, because of external reporting requirements, they are able to extract what is needed, while protecting the integrity of the data they have access to (Webber \& Calderon 2015:157).

Despite the collaboration between QA and IR, the relationship can still be regarded as ad hoc. Often one-way communication of data from IR to QA is implied, while the scope of the data is limited to the requirements of a particular quality review. It also seems as if IR often excludes QA or that QA operates in isolation from IR.

The core functions of QA and IR seem to differ. QA focuses mainly on quality reviews of different units of analyses, i.e. institutions, programmes and/or modules. Conventional IR is the sum of all activities directed at empirically describing the full spectrum of functions (educational, administrative and support) which are used for the purposes of institutional planning, policy development and decision-making (Saupe 1990 in Calderon \& Mathies 2013:81). Although the focus of IR has shifted over the past 20 years from reporting to active involvement in strategic positioning of the institution (Calderon \& Mathies 2013:81), references to IR do not necessarily include QA. Thus, there is only limited evidence of progress towards more strategic and integrated approaches.

The need for a relationship between QA and IR is accepted as a point of departure for this chapter, but the nature and scope of the collaboration and/or integration require further exploration. The purpose of this chapter is to explore the drivers, purpose, functions and maturity of QA activities in public higher education in South Africa; to describe the status of organisational structure and the location of QA units in public higher education institutions; and to explore the role and collaboration of QA and IR supporting decision making.

\section{Quality Assurance and Institutional Research}

\section{Historical overview}

The establishment and development of IR is linked to the significant expansion of education after World War II, which resulted in the concerted effort to plan and manage resources efficiently, particularly in education and higher education. Globally, the practice of IR has risen out of the mandate for institutions to report statistical information to governments and has evolved further as the reporting and accountability requirements increased (Calderon and Mathies 2013:81-82).

Compared to IR, formal QA systems in higher education are a fairly new phenomenon, despite older systems in the United Kingdom and the United States dating back more than a century. Widespread adoption of the current system in the United States, however, only occurred in the 1950s and 1960s (Kinser 2014:55). 
In South Africa, the first national unit of this nature was established as recently as 1995 (Jacobs, De Bruin and Jacobs 2013:20). This was a result of planning, funding and quality assurance being identified as the three mechanisms used to steer the South African HE system towards the national goals set out in the 1997 White Paper on Higher Education Transformation (Ministry of Education, May 2006:6). The Council on Higher Education (CHE), which was established in May 1998, was given executive responsibility for sectorwide quality assurance and promotion which it discharges through a permanent committee, namely the Higher Education Quality Committee (HEQC) (Department of Education 1997:Section 2.35). The HEQC is mandated to promote quality assurance in higher education, audit the quality assurance mechanisms of higher education institutions and accredit higher education programmes (CHE 2004:3).

The HEQC developed a framework and criteria to enable institutions to introduce QA. The purpose was to assist with making judgements regarding the quality of higher education and thus to enable the HEQC to develop appropriate policies and procedures to fulfil its mandate (CHE 2004:3).

In the South African context, quality is defined severally. It implies fitness for purpose, providing value for money, achieving effectiveness and efficiency of provision, enabling transformation and meeting the requirements of social development and economic and employment growth (CHE 2004:3). It is clear from the literature that there is no "universal definition" that would suit different contexts and all types of institutions (Schindler, Puls-Elvidge, Welzant \& Crawford 2015:3). Definitions of quality around the world are determined by various aspects such as culture, current developments in the sector and the policy framework of the country.

References to quality enhancement (QE) began to appear internationally after 2000. In South Africa, the national Quality Enhancement Project (QEP) commenced in January 2014 to improve student success in the sector. Four focus areas for the QEP were identified, namely the enhancement of academics as teachers, the provision of student support and development, the improvement of the learning environment and the management of enrolment (CHE 2014:1-25).

\section{Driving forces for the expansion of $Q A$}

Kinser (2014) states that several global trends served as driving forces for the expansion of quality assurance. These trends are also observable in South Africa, in particular after the advent of democracy in 1994.

Firstly, the diversification of HE systems introduced a variety of institutional types. Governments are increasingly holding institutions accountable, especially with reference to broader political and economic demands, to offer a variety of programmes to meet employability demands.

A second driving force was the emergence of a private higher education sector that was diversified, small and had a single purpose. This raised awareness about quality. These private institutions, in some instances, lacked academic credibility because they could not 
claim a historic reputation and had no record of research accomplishments. QA activities were instituted to determine the legitimacy of these private institutions.

Thirdly, increasing demand for higher education forced institutions to provide evidence of quality to students and policymakers to define institutional values and ensure relevant offerings. The danger of these demands is that they can warp the mission of HE. QA provides a counterweight to these market pressures.

A fourth trend that necessitated QA is internationalisation. Countries determine which educational products will be offered within their own borders, but increased movement of universities into foreign regulatory environments presented new quality challenges.

During the first cycle of QA in South Africa driven by the CHE (2004 - 2012), higher education institutions underwent comprehensive quality audits to evaluate and strengthen QA processes in their three core functions of teaching and learning, research, and community engagement. Many institutions customised their QA processes based on the national framework and criteria. The institutional audits also led to the establishment of QA units in many South African higher education institutions. A few years down the line, it is not clear to what extent these extensive QA activities impacted on lasting and sustainable QA practice in institutions. It might be assumed that there would have been development and maturing since then, but the chapter will explore how deeply integrated QA really is in institutions and in the sector. It will also examine the nature of its links with IR activities.

\section{Limitations of the study}

The literature review underpinning this chapter focussed on principles and historical practice pertaining to QA and IR in different international contexts. The empirical study we conducted covered only the South African public higher education sector. The findings are therefore mainly applicable to this particular context and preclude generalisation.

In this chapter, references to IR exclude QA unless otherwise stated. References to QA units include related organisational units such as planning and IR. The use of the designations QA manager or director is interchangeable with any other designations referring to heads of QA units.

\section{Research aim and methodology}

The key element explored during the research for this chapter was QA as it is manifested in different institutional contexts with regard to its functions, staffing, reporting lines, maturity and, specifically, its collaboration with IR. A further element is the roles both QA and IR units play in supporting decision making.

The literature review of QA and IR provides a theoretical framework for the chapter, while the empirical study elaborates further on the key elements in QA units in South African public higher education institutions.

Empirical data were collected by means of two pilot interviews with QA managers at two different institutional types. The purpose of the interviews was to inform and refine 
the research questions. An electronic questionnaire was then distributed to the heads or directors of the QA units of 21 public higher education institutions in South Africa with well-established QA units. This included traditional universities, universities of technology and comprehensive universities, i.e. the main institutional types in South Africa. Fifteen responses were received. The reports on the empirical findings are integrated with the literature review and discussed in this chapter.

\section{QA functions and competences}

Institutions and programme offerings obtain public legitimacy and academic credibility by means of explicit and visible QA policies and procedures. This also relates to organisational support, resources and infrastructure taking into account the institutional purpose and strategic direction. It is therefore inappropriate to compare QA practices across different institutional types (Kinser 2014:60). Programme quality can be determined internally (by the institution) or externally (by the government or a professional body), based on peer evaluations. It involves evaluating the programme against the standards and outcomes set by professional bodies, governments, or industry (Kinser 2014:59). Programme quality is to some extent more comparable between institutions.

The first aspect to be highlighted is the insight gained from the research regarding the naming conventions of QA units. This is deemed important as it does provide some insight into the emphasis of quality in the institution, as well as the prominence of combining it with other functions.

\section{Naming conventions of $Q A$ units}

Institutional contexts inform names, reporting relationships and functions of QA units. Leimer (2012:56) studied 30 IR and 30 Institutional Effectiveness Offices in the USA, and found that the functional components typically included institutional research, programme reviews, service and support unit reviews, programme accreditation and re-accreditation and strategic planning. In some cases, institutional budgeting, financial planning and analysis, space planning and programme development were also prominent.

Of the 15 South African institutions that responded to the survey, the names of 12 units relate to quality promotion, quality enhancement or quality advancement. This indicates a strong focus on the quality process and the main focus or purpose of the unit. It also suggests a developmental approach to QA, as opposed to an accountability focus. There is a sense that units wish to present themselves as constructive and contributing to improvement activities, rather than merely assisting with compliance processes.

Two institutions combined quality and planning in their names, and one institution combined quality and management information, indicating close relationships between these functions. This was, however, only observed in these isolated instances, which can be viewed as showing some level of integration taking place. 


\section{Functions of $Q A$ units}

An open-ended question in the survey requested respondents to indicate the core functions of the QA office. A qualitative analysis and clustering of responses resulted in the following table depicting the core functions:

Table 1: Core functions of QA units

\begin{tabular}{|l|c|}
\multicolumn{1}{|c|}{ Categories of functions } & Number of QA units \\
\hline Establish and enhance a quality culture & 10 \\
\hline $\begin{array}{l}\text { Internal and external reviews (included programmes, academic departments, } \\
\text { service and support units, national reviews) }\end{array}$ & 8 \\
\hline Monitoring and guidance related to broader improvement activities & 8 \\
\hline Institutional alignment with national QA processes & 7 \\
\hline Internal alignment with strategic planning processes & 7 \\
\hline $\begin{array}{l}\text { Research, both QA-related and conventional IR (e.g. surveys, impact studies, } \\
\text { environmental scanning) }\end{array}$ & 7 \\
\hline Programme development, approval and amendments & 6 \\
\hline Capacity building & 6 \\
\hline National Quality Enhancement Project (QEP) & 5 \\
\hline $\begin{array}{l}\text { Funding related activities such as Higher Education Management Information } \\
\text { System (HEMIS) and/or Programme Qualification Mix (PQM) }\end{array}$ & 4 \\
\hline Supporting institutional governance & 4 \\
\hline Academic planning and/or enrolment management & 4 \\
\hline
\end{tabular}

Some functions listed in the responses apply to only one institution and are thus not included in the table, for example, student ombudsman or verification of qualifications.

The main categories of functions of QA units listed in Table 1 can be integrated into four themes:

i. Quality culture

Ten QA units see their task as establishing and sustaining a quality culture in the institution, i.e. "to promote a culture of continuous improvement". References were made to a QA "regime", QA management, a framework and system that include policies and procedures, and "a responsive quality management policy framework".

ii. Monitoring and review

The next two categories in table 1 above (8 respondents each) can be combined as broadly focussing on continuous review activities and monitoring of progress. These functions also include the development of improvement plans, and the monitoring and identification of gaps in the institutional QA system.

iii. Alignment

External and internal alignments (7 QA offices in each case) are described as a key feature. This includes alignment with national quality imperatives, and with the objectives, mission and vision of the institution. 
iv. Research

The QA units conduct various forms of research. These range from conventional IR activities such as surveys, collating external reporting trends and desktop research to researching national and international developments in quality enhancement. According to the respondents, this also includes gathering and analysing information to identify good practices.

In addition to the findings above, a number of observations are noteworthy. Six of the QA units (40\%) support programme development, approval and amendments. Six of the QA units identified staff and capacity development as core functions. In a separate question, all fifteen respondents indicated that they develop the competencies of their own staff members (although it is not always regarded as a core function of the unit). QA units indicated that such capacity building is done both internally and externally. Only five QA units mentioned involvement in the national QEP project at institutional level. This might be because of the current QEP themes being regarded as closely aligned to academic development. Only four of the 15 QA units are involved in the management of databases, e.g. the PQM, HEMIS and enrolment management, as these functions are regarded as conventional IR activities.

\section{QA intelligences required}

In this section we report the findings from the survey using the three tiers of organizational intelligences distinguished by Terenzini (1993:1-10, 2013:137-148). These are deemed to be equally important and interdependent, and needed by staff working in these units. The intelligences are:

\section{Tier 1: Technical/analytical intelligence}

This category provides for two types of intelligences. Firstly, factual knowledge of IR data, e.g. number of students, staff, financial and facilities information, terminology, coding conventions, familiarity with institutional structures, basic institutional data on applications, admissions, registration, to name a few. Secondly, analytical, methodological skills and competencies such as research design, sampling, statistics, quantitative and qualitative research methodology, enrolment projections and forecasting, academic workload, library research skills, communication skills and technical computer skills.

This level of intelligence is regarded as fundamental and foundational, but without the next layer of intelligence, it has very little value.

The survey respondents were requested to identify the aspects of technical/analytical intelligences (i.e. the first tier) required by staff in their units. The percentages and actual numbers below indicate how many QA units identified primary skills required to function successfully in such units: 
Table 2: Technical/analytical intelligence $(n=15)$

\begin{tabular}{|l|c|}
\hline \multicolumn{1}{|c|}{ Technical/analytical intelligence } & Percentage and number of QA units \\
\hline Computer skills & $100 \%$ (15 units) \\
\hline Oral and written communication & $87 \%$ (13 units) \\
\hline Presentation skills & $87 \%$ (13 units) \\
\hline Familiarity with terminology (i.e. standard/basic/operational terms) & $73 \%$ (11 units) \\
\hline Familiarity with core policies and procedures & $67 \%$ (10 units) \\
\hline Quantitative research knowledge & $40 \%$ (6 units) \\
\hline Qualitative research knowledge & $40 \%$ (6 units) \\
\hline Library research and information skills & $27 \%$ (4 units) \\
\hline
\end{tabular}

QA staff members are often required to do presentations, conduct workshops, participate in discussions and proactively share knowledge (Knight 2015). Only six (40\%) of the QA units require qualitative or quantitative research knowledge and skills from their staff members. This can be linked to the broad research function fulfilled by the units. It could include document analysis (e.g. to identify QA trends, reports, policies, and national documents) rather than traditional research. It may also explain why library research was identified by only four (27\%) of the QA units.

\section{Tier 2: Issues intelligence}

This tier of intelligence requires knowledge of the major decision areas that institutions face: enrolment planning, academic workload, resource allocation, facilities planning, management and maintenance, programme and institutional planning, reviews, etc. Issues intelligence is required to resolve these substantive problems. It involves an understanding of the major categories of problems confronting institutional managers. Furthermore, it requires knowledge of how the institution functions and the political character of the decision-making areas. It thus includes the skill of how to work successfully with others in professional settings.

Leimer and Terkla (2009:45) emphasise the importance of social intelligence, namely, to be able to navigate complex social situations. This is regarded as a critical attribute. The extent to which this is an acquired skill is debatable, however, as it seems to be a skill that the individual can develop over time. Staff often find themselves in positions and situations where they must rely on negotiating and mediating skills. A high degree of social intelligence facilitates collaborative relationships.

In the survey respondents were asked to identify the aspects of issues intelligence that their staff need to perform their core functions. The percentages and actual numbers below indicate how many respondents identified these aspects as a primary requirement for QA staff: 
Table 3: Issues intelligence $(n=15)$

\begin{tabular}{|l|c|}
\multicolumn{1}{|c|}{ Issues intelligence } & Percentage and number of QA units \\
\hline Facilitate programme reviews & $93 \%$ (14 units) \\
\hline $\begin{array}{l}\text { Persuasion, compromise, prior consultation with opinion makers, } \\
\text { personal and professional courtesy }\end{array}$ & $80 \%$ (12 units) \\
\hline $\begin{array}{l}\text { Ability to identify the major issues/challenges/ and priority } \\
\text { decision areas }\end{array}$ & $47 \%$ (7 units) \\
\hline New programmes development & $47 \%$ (7 units) \\
\hline
\end{tabular}

The two top categories of issues intelligences required by QA staff are in line with the top core functions (namely, quality culture, programme reviews and monitoring, external and internal alignment). Programme reviews require knowledge of the process, as well as facilitation skills for consistent implementation. QA staff thus need a unique combination of skills and knowledge of processes and technical requirements, coupled with social and interpersonal skills.

\section{Tier 3: Contextual intelligence}

Contextual knowledge requires an understanding of the national and international higher education quality culture, but also at an institutional level. It includes the historical and philosophical evolution, culture, political structures and influences, decision-making processes and levels, and also the values and attitudes of the people at different levels. Terenzini (1993:5-4) refers to this tier of intelligence as "organizational savvy and wisdom". Demonstrating this level of intelligence also earns legitimacy, trust and respect.

The fifteen respondents identified the following aspects of contextual intelligences as a requirement to perform their primary functions.

Table 4: Contextual intelligence $(n=15)$

\begin{tabular}{|l|c|}
\hline \multicolumn{1}{|c|}{ Contextual intelligence } & Percentage and number of QA units \\
\hline Understanding values and attitudes of people at all levels & $67 \%$ (10 units) \\
\hline Understanding of national higher education & $60 \%$ (9 units) \\
\hline $\begin{array}{l}\text { Understanding institutional culture, including its history and } \\
\text { philosophical evolution }\end{array}$ & $53 \%$ (8 units) \\
\hline $\begin{array}{l}\text { Understanding institutional governance, decision-making processes, } \\
\text { customs and levels }\end{array}$ & $53 \%$ (8 units) \\
\hline Understanding of international higher education & $40 \%$ (6 units) \\
\hline
\end{tabular}

The intelligences listed in the table above can be labelled as "big picture thinking" or "strategic leadership". Leimer and Terkla (2009:45) emphasise the importance of strong leadership qualities, because of engagement with academics and leaders, as well as lower level staff, both on the academic and support side. Leimer (2012:49-50) noted the importance of the abilities to build consensus, negotiate, to communicate in non-technical language and to co-ordinate people and projects. The characteristics of such a leader include open-mindedness, flexibility, a capacity to listen, a commitment to learning and a sense of humour. It also requires building confidence and motivating others, creativity, team-building 
and problem-solving capacities. QA leaders are also required to demonstrate tolerance for ambiguity, patience and the abilities to educate, the ability to build trust and to use data to relay and present a compelling story.

Contrary to the first two tiers of intelligence, where there was a high rate of respondent agreement, the responses regarding the third tier of intelligence were more disparate. This could point to a less developed maturity of contextual intelligence in the South African QA environment.

The three kinds of intelligence may be mutually dependent and supportive, and all three forms contribute to an effective QA office, but are not always found in one individual (Terenzini 1993:1-10, 2013:137-148). This emphasises the need for an integrated IR unit staffed by practitioners with a range of skills and competencies.

\section{Maturity of $Q A$ units}

Volkwein (2008:16) posed the question whether IR can be defined or viewed as a mature profession. The same question can be asked of QA. The optimum level of maturity is the ability to contribute to the delivery of the institution's strategic objectives. Different maturity models emphasise different aspects of IR. Taylor, Hanlon and Yorke (2013:66-71) developed a possible maturity model, taking the multifaceted nature of IR into consideration. We maintain that this model can also be used to analyse the maturity levels of QA units. The model proposes the following broad functions: routine institutional management, strategy formulation, QA and quality enhancement (QE), marketing and competitor analysis. In the context of this chapter, the inclusion of both QA and QE is noteworthy.

The highest level of maturity (level five) is reached when the unit contributes significantly to the delivery of strategic objectives most effectively and efficiently. This level focuses on the implementation of an institution-wide, integrated and well understood QA system. In the South African context, the first cycle of institutional audits focused on the institutional quality systems - these audits, in other words, served as a driver to establish institutional QA systems. The consistent implementation of an institutional system across the institution, both on the academic and administrative side, remains a challenge.

Maturity level four is reached when an integrated system with standardised processes and regular and systematic feedback mechanisms to students and staff has been established.

The third level of maturity is deemed by Taylor et al. (2013:70) to be "semi-maturity". It is characterised by more comprehensive processes (e.g. more regular reporting) compared to an ad hoc approach as in level two), but not as systematic and formalised (as in level four).

Maturity level two is characterised by ad hoc and/or isolated QA processes, some better than others with some inconsistencies across the system.

QA units operating at maturity level one demonstrate a complete absence of QA functions and thus no implementation is possible. 
Respondents had to indicate which of the QA and QE functions (according to the levels in Taylor, Hanlon and Yorke's (2013:66-71) proposed maturity model) are facilitated by the QA unit as core functions. Table 5 below provides an analysis of the QA and QE functions in terms of the 5 levels of the proposed maturity model of Taylor et al. (2013:66-71).

Table 5: $\quad$ Levels of maturity of $Q A$ units $(n=15)$

\begin{tabular}{|l|l|c|}
\hline \multicolumn{1}{|c|}{$\begin{array}{c}\text { Level of } \\
\text { maturity }\end{array}$} & \multicolumn{1}{|c|}{ QA function (includes QE) } & $\begin{array}{c}\text { Percentage and } \\
\text { number of institutions }\end{array}$ \\
\hline \multirow{2}{*}{$\begin{array}{l}\text { Level 5 } \\
\text { High level of } \\
\text { maturity }\end{array}$} & Institutional quality management framework adopted & $87 \%(13)$ \\
\cline { 2 - 3 } & $\begin{array}{l}\text { Implementation of a formal quality management system, } \\
\text { based on closing the quality loop }\end{array}$ & $87 \%(13)$ \\
\cline { 2 - 3 } & Predictive data analyses & $13 \%(2)$ \\
\hline \multirow{2}{*}{$\begin{array}{l}\text { Level 4 } \\
\text { Maturity }\end{array}$} & Evidence of professional and regulatory accreditation & $87 \%(13)$ \\
\cline { 2 - 3 } & Systematic programme reviews schedule and targets & $100 \%(15)$ \\
\cline { 2 - 3 } & Regular feedback mechanisms to all staff & $87 \%(13)$ \\
\cline { 2 - 3 } & Regular feedback mechanisms to students & $33 \%(5)$ \\
\hline \multirow{2}{*}{$\begin{array}{l}\text { Level 3 } \\
\text { Semi-maturity }\end{array}$} & Multi-dimensional reporting on programme quality & $100 \%(15)$ \\
\cline { 2 - 3 } & Systematic reviews of student experience & $40 \%(6)$ \\
\cline { 2 - 3 } & Regular reporting to stakeholders & $80 \%(12)$ \\
\hline \multirow{2}{*}{$\begin{array}{l}\text { Level 2 } \\
\text { Inconsistent } \\
\text { maturity }\end{array}$} & Some evidence of student feedback mechanisms & $67 \%(10)$ \\
\cline { 2 - 3 } & Limited/ad hoc programme reviews & $100 \%(15)$ \\
\cline { 2 - 3 } & Reporting to stakeholders on QA & $87 \%(13)$ \\
\hline \multirow{2}{*}{$\begin{array}{l}\text { Level 1 } \\
\text { Immature }\end{array}$} & No evidence of QA system and thus not implemented & $0(0 \%)$ \\
\hline
\end{tabular}

We will now discuss this analysis to identify the level of maturity of QA offices in SA, keeping in mind that only institutions with well-established QA units were included in the survey.

\section{Level 5: Highest level of maturity}

Thirteen of the 15 QA units indicated that they are involved in the development and implementation of an institutional quality framework. As explained above, the national institutional audits conducted by the HEQC have focussed on quality arrangements, mechanisms and procedures. This focus included evidence of a cyclical approach from planning to implementation, and subsequent monitoring and review of the implementation of improvements. The extent to which these institutions have internalised QA as part of their strategic and operational management was not explored. The adoption of an institutional quality management system, however, probably refers to a system based on the national approach introduced by the South African CHE. Thus, it is evident that after a number of years, institutions are still merely responding to quality requirements as prescribed or expected by government agencies. The challenge is often to close the loop that takes the process through to improvement and implementation. This is when quality assurance activities become integrated and embedded in the culture and strategy of the institution. 
The fact that the majority of institutions indicated that the system is being implemented is a redeeming factor; institutions have matured beyond the level of demonstrating evidence of quality systems on paper only.

Predictive analysis was identified as a core function by only two QA units, while eight indicated that the responsibility for this resides with another unit in their institutions, and five units indicated that this is not done at their institutions. This may be regarded as a more traditional IR function and could therefore be the reason why the majority of IR units did not identify this as a function.

\section{Level 4: Maturity}

All the respondents (i.e. 100\%) indicated that they implement systematic programme reviews and that they support professional body reviews. This relates to the core functions listed in Table 1.

Regular feedback mechanisms to staff seem to be in place at the majority of institutions (i.e. $87 \%$ - see the section on reporting lines). It is, however, evident that regular feedback to students remains challenging - only five QA units provide regular feedback. In many countries around the world, feedback from students is used to assist them with institution and programme choices. At this stage it is assumed that pricing and financial obligations, location, etc. are the major contributors to student choices. Thus, it is not clear why institutions can currently make claims of "value for money", as there is no real evidence available to substantiate these claims.

\section{Level 3: Semi-maturity}

All fifteen QA units do multi-dimensional reporting on programme reviews. Twelve of the 15 institutions do reporting to other stakeholders. The matter that seems to be neglected by the majority of institutions is the systematic review of student experience. This may be a function (i.e. a survey) undertaken by the IR office, but quality reviews are conducted by only six of the fifteen institutions.

\section{Level 2: Inconsistent maturity}

All the QA offices indicated that ad hoc programme reviews are conducted (see level two above), even though they have a systematic schedule for programme reviews with clear targets in place (see level four above). QA units seem to be versatile enough to accommodate ad hoc programme reviews as required by the context.

\section{Level 1: Immature}

None of the QA units operates at this level.

To summarise, mapping the maturity of the QA units in terms of the levels proposed above has proved to be a complex process. The findings on the maturity levels of QA units at South African universities reveal that there are challenges in the system, even though the majority of QA units assist with the development and implementation of institutional 
quality management systems. These QA systems include systematic student feedback and integrated data. Quality reviews seem to be restricted to programmes and there are not many indications of alternative units of review (such as at the level of a school or a faculty).

The purpose of this discussion was not to peg QA units at a particular level, but rather for QA units to consider the challenges in addressing their core functions. It may also provide institutions with an opportunity to take a critical look at their QA systems with regard to the core functions listed above.

In this section we have provided an overview of the core functions fulfilled by QA units in public higher education institutions in South Africa. The naming conventions used for QA units clearly focus on quality promotion, enhancement and advancement, while the functions primarily focus on quality culture, programme reviews and monitoring, and general guidance relating to quality improvement. We have also outlined the competency profile of staff in QA units. The analysis of the maturity levels of QA units in South African universities highlights the challenges that exist at institutional level, but it also indicates some evidence that QA units play a role in the development and implementation of institutional quality management systems.

\section{Organisational structure}

The focus now shifts to the structure of the QA units. The previous section on naming conventions should be kept in mind when matters such as staffing and reporting lines are considered.

\section{Staff complement}

The staffing of QA units clearly depends on the size of the institution, the scope of its academic offering and the role expected of the QA unit.

Leimer and Terkla (2009:47) state that higher education institutions can best be served by what Volkwein (2008:13-14) referred to as a "professional bureaucracy". This refers to the size and formal structure of the office, where staffing includes at least four professionals, staff members with a wide range of credentials and experience in the field. More than one staff member should hold a doctorate and have more than 10 years of experience in the field. Included in this bureaucracy are student/graduate assistants and analysts who are new to the field. This arrangement provides the capacity to conduct sophisticated research (Leimer and Terkla 2009:19-19). This is a typical QA or IR office structure in the USA (Calderon \& Webber 2013).

We provide data in the following section on the staffing in QA units to provide a national overview of QA structures. It is interesting to consider the similarities and differences with the staffing profile of the IR units in South African universities, as reported in Chapter 4). 


\section{Appointment of staff}

Leimer and Terkla (2009:110) identified sources of dissatisfaction among staff in QA units (in the USA, but there is also evidence that it is wider than the USA) to include limited career progression, development and promotional opportunities, stressful job demands and excessive workloads. Managers/heads often have a limited team and extensive reporting demands, limited access to information systems, data integrity issues and lack of access to decision makers.

In South African universities, a number of issues have an impact on staffing and the ability to attract suitable practitioners for QA units. These include the appointment type (e.g. category of appointment being administrative, professional or academic), lack of clear career pathing and promotion opportunities, the skills sets required, transformation agendas and the availability of so-called scarce skills, and less competitive salaries paid by universities in general. Staff members are often recruited from the academic sector, which affords different promotion opportunities or benefits. In some cases, there are requirements for staff to retain their academic involvement and to continue to conduct research, but mostly this would be in relation to quality assurance and not specific to the discipline they came from or were transferred from. There is also a perception that QA staff are less qualified or less knowledgeable, which in turn can result in a credibility issue.

In the Figure 1 below, information on the categories of appointments across institutions is provided, divided into academic, service and support (non-academic or professional) or administrative categories.

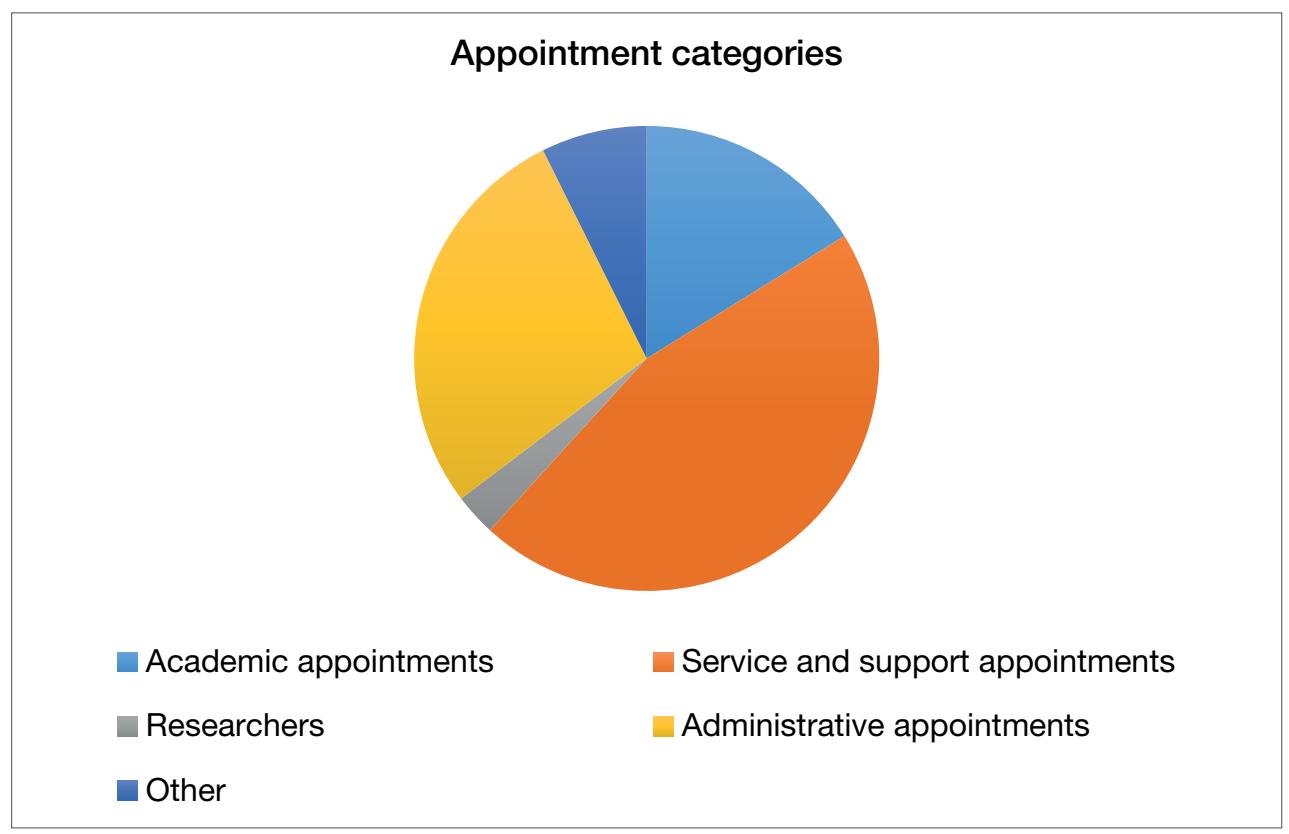

Figure 1: Appointment categories 
Sixteen percent of the respondents have academic appointments, while the majority, (i.e. $45 \%$ ) are service and support appointments. One might question the suitability, in terms of performance and credibility, of each type of appointment for QA work. One argument is that a QA unit needs both types of appointment to provide support across the institution. This is a matter that could be debated at a national forum for QA staff, but, if it is, individual institutional contexts should be taken into consideration.

\section{Qualifications}

Knight and Leimer (2010:110) state that experienced IR professionals are difficult to find and that the development of expertise involves long periods of training. This could perhaps suggest that there is no formal educational route or specific qualification which prepares the IR professionals or that the route is very limited. Even experienced staff need time to familiarise themselves with the idiosyncrasies and systems of institutions to which they are appointed. Because of the small size of the units, the loss of an employee severely reduces capacity. Extended periods of time are needed for recruitment and training.

In South Africa, there are no formal qualifications in either QA or IR in higher education. In Figure 2 below, the diversity of qualifications of staff members in QA units is illustrated.

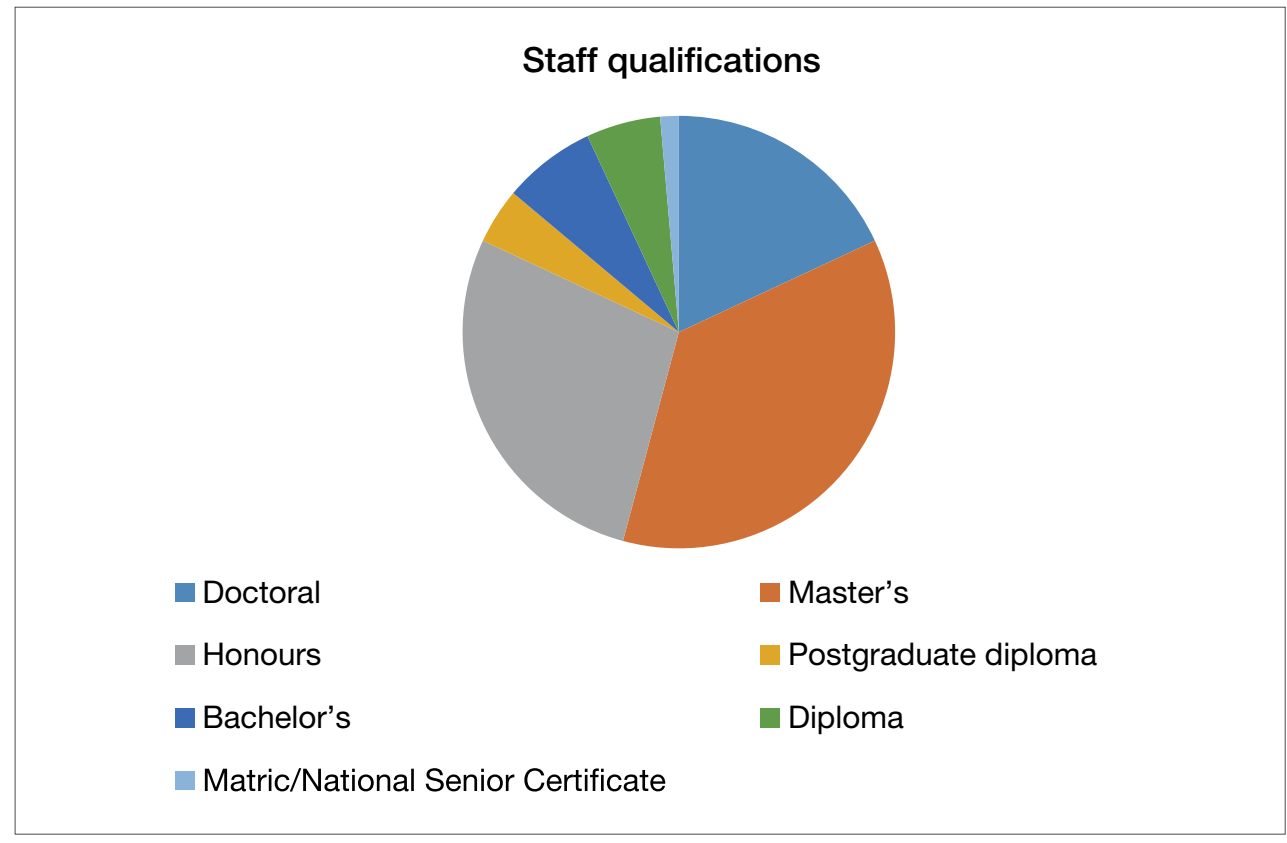

Figure 2: Staff qualifications

The majority of the existing staff complement in the 15 institutions, i.e. $82 \%$, possess postgraduate qualifications: $36 \%$ have obtained a Master's degree; $28 \%$ an Honour's degree and $18 \%$ a doctorate. Although this is commendable, it does not guarantee academic training in QA. This could be interpreted as lack of formal evidence of skills and knowledge in the 
field of QA. These skills are often acquired over a number of years, through experience and engagement with peers and through self-study and exploration, and not through formal education.

To elucidate the matter, respondents were also asked to indicate the fields of specialisation of their staff members. Across the different categories of qualifications (as in Figure 2 above), the majority of staff members specialised in education/higher education, i.e. a related field of specialization. This reiterates the statements above on the lack of formal training in QA. Other disciplines include a broad spectrum, for example Theology, Social Science, Hospitality, Linguistics, Management, Human Resources, IT).

\section{Duration of service}

Figure 3 below presents the current staff members' years of service in a QA unit:

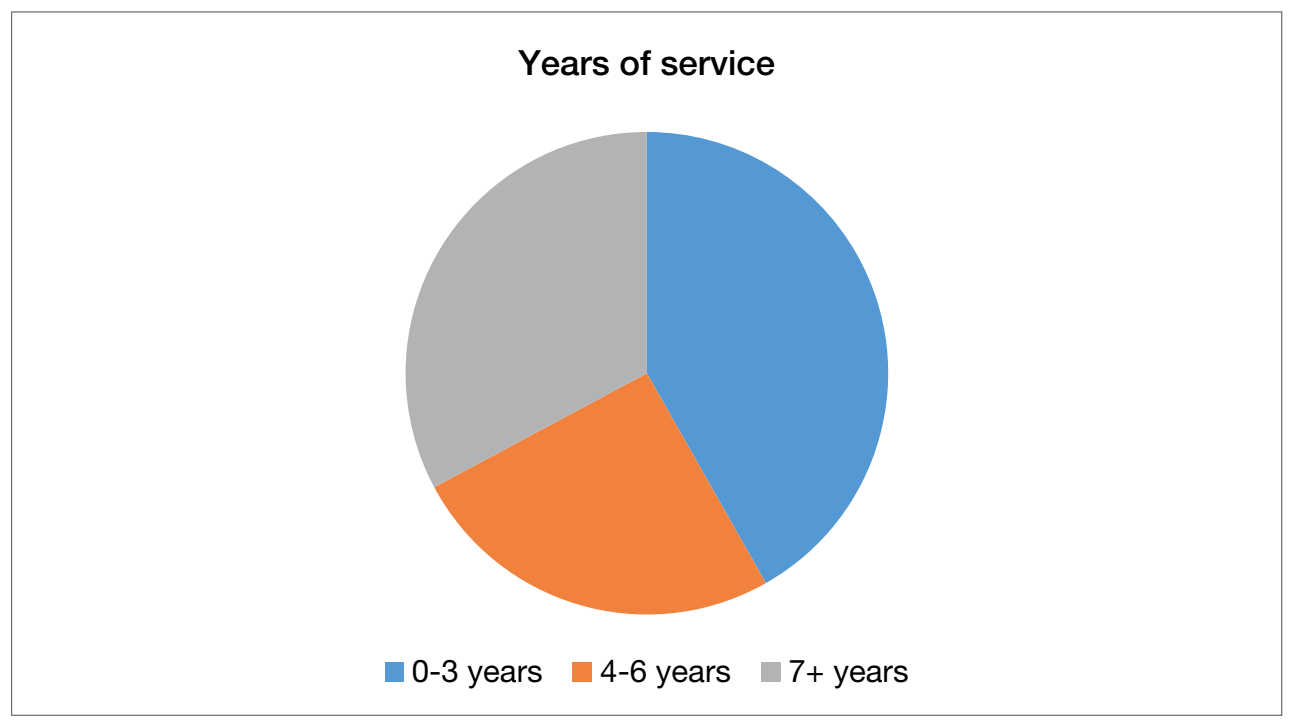

Figure 3: Years of service

Staff members with less than 3 years' experience in the QA field, make up $42 \%$ of the total number of staff members. They can be regarded as the group that is still acquiring the necessary knowledge in skills in the QA domain. The fact that the experienced group of staff members with more than 3 years' experience in the QA field (i.e. the $25 \%$ with 4 to 6 years' experience and the $22 \%$ with more than 7 years' experience) make up the majority group and can be regarded as a redeeming factor.

Reflecting on the above staff-related data, we can come to a number of conclusions. QA is a relatively new practice in South African higher education, and, with the absence of formal qualifications, this poses a challenge for finding sufficiently experienced staff. The large numbers of QA staff specialising in education/higher education is encouraging, but this might be owing to the migration from the academic sector or to the progression and 
maturity of QA and IR as disciplines over the last decade. Migrating from the academic sector is positive in the sense that it provides an academic background and understanding, but it is often restricted to a particular discipline, field or department. QA and IR require professionals to function within the broader institutional context and thus move beyond their original discipline.

As we have noted, the variety of qualifications and fields of specialisation can be interpreted as a result of migration from other disciplines. QA units often employ staff members with related qualifications in similar fields, which could include fields within education. Because of the lack of available formal programmes in QA and IR specifically, it is not often that a staff member can be recruited directly after completing these studies. Related studies can serve as an advantage, as many fields supplement IR and QA, it is, however, often also a hindrance to staff because of discipline-specific approaches to QA. For instance, business environments have a particular definition and approach to QA which is often not transferable to higher education. It could also demonstrate that QA is a scarce skill, and that staff are employed on the assumption that they will acquire the new and necessary skills on the job.

The relatively high number of staff members with doctorates is probably an indication of migration from academic disciplines and, in some cases, from struggling academic disciplines (such as Theology). Very few of these doctorates would be focused on, or specialised in, QA.

The fact that $18 \%$ of the current staff component have been working for more than 7 years in quality related functions can be interpreted as providing stability to this domain. The staff members in the 4-6 years' bracket can be interpreted as the 'next generation' of QA professionals, which is both encouraging and concerning as this is not deemed to be sufficient in the context of the rising quality challenges in the South African context. The 0-3 year's bracket can be regarded as the group where the biggest staff turn-over can be expected (Materu 2007). There is, in other words, a high mobility in the early employment years.

\section{Organisational and reporting lines}

The reporting relationships vary widely across institutions. Some units report to executive management levels, whereas other units are lodged within an institutional unit with lower level reporting lines. Various reporting relationships are based on the historical involvement of particular staff members, in some cases the unit has matured and evolved, but the reporting line has not been adjusted accordingly. QA units should be positioned to inform the relevant decision-making process directly. It is also clear that the variety of responsibilities and functions of the office make the placement and reporting line more difficult. IR units often represent higher levels of reporting because of external reporting requirements, which are related to funding and compliance.

Table 6 below provides an overview of the reporting lines of the QA units that responded to the survey: 
Table 6: $\quad$ Reporting lines $(n=15)$

\begin{tabular}{|l|c|}
\multicolumn{1}{|c|}{ Direct reporting lines } & Number of institutions \\
\hline $\begin{array}{l}\text { Senior/Executive Director to DVC: Academic } \\
\text { (Teaching and Learning) or VC }\end{array}$ & 6 \\
\hline Executive Assistant to VC & 1 \\
\hline Registrar & 2 \\
\hline DVC: Academic (Teaching and Learning) & 3 \\
\hline VC & 3 \\
\hline
\end{tabular}

The majority of the QA units have multi-layered reporting lines. The unit reports individual academic departments and faculties, and to academic support units on programme reviews. It reports at institutional level to one or more Senate committees. It also reports to a member of the senior management team ( 6 of the respondents indicated that they report to a deputy vice-chancellor or directly to the vice-chancellor).

The versatility required when reporting to different 'clients' (often with different purposes) is challenging. It can be linked to the need for experienced staff with an understanding of the institutional role players, i.e. contextual intelligence which is often found only in the head of the QA unit (as discussed above).

Also, in some instances, the QA unit reports to a different line manager to the IR unit, and thus the two functions often reside in different portfolios. Some units also have a higher reporting line than others, for instance, the IR unit will report to the VC while the QA unit reports to the DVC. This means QA units are further removed from decision makers, and the complexity is increased by having the portfolios with different reporting lines. Reporting to the Registrar or Deputy Vice-Chancellor Academic (or Teaching and Learning) or directly to the Vice-Chancellor seems to be most common.

\section{Informing the institutional decision}

The QA directors gave different responses when asked to indicate whether their reports and/ or proposals inform decision-making, and if so, how. The following categories of responses were received $(\mathrm{N}=12)$ :

Four of the QA units indicated that they deem their reports to be valuable to faculty level functions and that they are being used to inform operational plans. They do not, however, feel that the reports impact on the strategic direction of the faculty or the institution. Their reports serve at a Senate committee or equivalent where input is obtained, which, in turn, makes it transferable to informing policy development. Another QA office indicated that their QA reports (as well as IR reports) are "central to decision-making processes in the institution". This statement, however, does not indicate that the QA and IR reports are integrated.

Four institutions qualified the impact of their reports/proposals by indicating that it depends on the staff or layers of management involved. Some management staff members are more influential than others, and some units or portfolios or personalities are taken more seriously 
than others. If the reports or proposals serve at executive level, then the impact is usually more significant, whereas, if there is no exposure of the content at a higher level of the institutional management structures, it can be viewed as not having impact or not being taken seriously.

Comments from four QA units indicated that their reports do not have impact, as the issues that require decisions remain unresolved. This then impacts on efforts to promote a quality culture. They also felt that the reports are often merely noted at Senate level and not translated to other parts of the institution. Many QA units also noted that they are not involved in the governance structures that consider the reports. In contrast to this, one institution claims that their reports are taken into consideration when high level decisions are taken, and they indicated integration between QA and IR. Collaboration/integration is discussed in the next section. The impact of QA reports at faculty level is limited and varies between faculties. Their use depends on the individual deans and line managers, and often on the relationship between them.

Three institutions provided descriptions of their reporting processes without referring to the impact of their reports on the decision-making process. They were excluded from the analysis above.

\section{Integration of QA and IR}

IR and other support units, such as QA, may provide data, but this is not always immediately usable or user-friendly nor is the application of the data self-explanatory. Data must be converted into information and institutional knowledge through analysis and interpretation. We recommend that responsibility should be allocated for applying it in the context of the institutional goals. To make it useable and understandable, data should be disseminated in multiple formats appropriate to the particular stakeholders and audiences, to help inform recommendations and planning.

The expectations of institutional executive managers are that IR units (including QA) should be pro-active. Leimer (2012:47) states that IR and QA staff, in an unintegrated structure, often experience their roles in a narrow way and as too junior to be involved in and inform strategic discussions. She sees the integrated model as a solution to a need for change in the HE sector that exceeds the current, more conventional capabilities. Integrated IR and QA units take on progressive leadership roles, educate staff and advocate the use of evidence in decision-making. They bring knowledge of, and provide HE expertise in, trends affecting their institutions through presentations, reports and discussions. Likewise, Calderon and Mathies (2013) talk of the importance of IR and QA, as integrated entities, being actively involved in the strategic positioning of the institution. The contents of the reports produced by these practitioners inform the goals, vision and mission, and help to ensure alignment.

Integrated functions do not necessarily mean that QA and conventional IR units are integrated into one office or organisational unit. This arrangement can also be achieved in a more decentralized manner, but with focused collaboration. Collaboration can be achieved in various ways, e.g. an integrated approach to governance or unique institution-specific mechanisms to strengthen these connections across separate units. 
In some institutions, both IR and QA related matters serve as items on the agenda, but in other cases a specific committee or working group is created to connect the two functions and to enhance integration between them.

In the survey, respondents were asked to respond to the list of statements related to the integration of QA and IR functions. The statements in Table 7 below are specifically focused on programme reviews, to ensure a common understanding and familiarity of application. The results are reflected in Table 10 below.

Table 7: Interaction between $Q A$ and IR $(n=15)$

\begin{tabular}{|c|l|c|c|}
\hline $\begin{array}{c}\text { Item } \\
\text { number }\end{array}$ & \multicolumn{1}{|c|}{ Integration between QA and IR } & Yes & No \\
\hline (i) & QA and IR are housed in one division or unit & 10 & 5 \\
\hline (ii) & QA and IR have the same reporting lines & 12 & 3 \\
\hline (iii) & QA and IR agree on the role of IR in programme reviews & 6 & 9 \\
\hline (iv) & IR is involved in programme reviews by providing data to inform the process & 10 & 5 \\
\hline (v) & IR is involved in site visits from regulatory bodies & 4 & 11 \\
\hline (vi) & IR is involved in the formulation of the programme review report & 4 & 11 \\
\hline (vii) & $\begin{array}{l}\text { IR is involved in the development of the quality improvement plan [following } \\
\text { recommendations from the review panel] }\end{array}$ & 3 & 12 \\
\hline (viii) & IR is involved in monitoring progress of the improvement plan implementation & 2 & 13 \\
\hline (ix) & QA submits annual report(s) on the outcomes of programme reviews & 14 & 1 \\
\hline (x) & IR is involved in the development of the report(s) on the outcomes of programme reviews & 4 & 11 \\
\hline (xi) & Programme review and IR reports are integrated & 7 & 8 \\
\hline
\end{tabular}

Reflecting on the data on the integration of QA and IR leads to a number of conclusions, which can be summarized as follows. The practice of submitting annual reports seems to be widely spread across the institutions. There are indications that the majority of the QA units are closely located to the IR units in terms of organisational structure and reporting lines. It did become clear, however, that this does not imply close collaboration between the functions or did not necessarily result in an integrated approach. The majority of the QA units seem to be independent as they have specific core functions that differ substantially from the conventional IR units. Self-evaluation reports are a key element of programme reviews and a core function of QA units. IR data, e.g. on student success, informs these reports. Reporting lines and levels do not, however, support integration. The interaction between QA and IR seems to be informal or assumed, because no formal arrangements seem to exist. There seems to be no mechanism or mutual platform available to sustain integration between QA and IR functions. Items (v) to (viii) (in Table 7 above) address typical QA functions; ad hoc IR involvement is relatively arbitrary. In considering items ( $x$ ) and (xi), there seems to be an integration of reports subsequent to independent reporting activities, thus indicating collaboration between IR and QA units to a limited extent. This could be interpreted as superficial collaboration or assumed collaboration, but no systemic integration of QA and IR with a significant impact on decision-making. 


\section{The involvement of QA units in decision-making}

QA units in the survey were asked to explain their involvement in decision making in their institutions. Three categories of responses were identified, which are categorized as a) No involvement, b) Involved and c) Indirectly/some involvement.

No involvement: Seven of the 14 QA units indicated no involvement in decision making. Some indicated that they may be consulted, but no formal involvement is facilitated or required.

Involved: Four QA units indicated that they are involved in decision-making through management structures.

Indirectly/some involvement: Three QA units qualified their involvement, i.e. that it is determined by the relevance/suitability of the issue or matters at hand or through informal engagement with their line manager. This indicates that they are more dependent on the nature of relationships.

\section{Conclusions and recommendations}

We have provided an overview in this chapter of the status of QA units, and their structures and functions in South African higher education institutions. The survey of QA units in South Africa was informed by literature and research on QA and IR. The empirical data focused firstly on QA units referring to naming conventions, core functions, required competences, maturity and organisational structure. The focus then shifted to the collaboration between $\mathrm{QA}$ and IR and their respective roles in decision-making processes.

Our results indicate that the majority of units refer in their names to quality enhancement, promotion and advancement, signifying a clear focus on quality matters in institutions. The majority identified their core functions as the establishment and enhancement of a quality culture in the institution; they were engaged in monitoring and reviews, and in guiding improvement plans and activities. QA practitioners require various competencies, such as personal computer skills, oral and written communication and presentation skills (i.e. technical intelligences), knowledge of programme reviews and people skills (i.e. issues intelligence). With reference to contextual intelligences, a deeper understanding of the people they work with is needed. The rest of the contextual intelligences can also be labelled as "big picture thinking" or "strategic leadership". This is, however, not embedded in all QA related structures. With reference to maturity, the majority of institutions are supported by QA units to implement a quality management system or framework, but the system remains challenging, specifically with regard to systematic student feedback, integrated institutional data and systematic reviews of student life-cycle experiences.

In the section on staffing, the lack of formal qualifications was highlighted and reflected in the diversity in the expertise of QA practitioners (although education/higher education was identified as the main field of expertise). Reporting lines vary across institutions with multi-layered reporting lines implemented by most of the institutions. The impact of QA reports and activities also varies across institutions, with the majority claiming little or inconsistent impact. 
Taking into consideration that QA is a relatively young profession in South Africa, there are a number of challenges across institutions, such as the absence of formal qualifications and a diversity of fields of specialisation and conditions of appointment of staff. Because of the absence of formal qualifications in QA, all QA units should address the necessary development of QA staff to build capacity, i.e. not only the required intelligences, but also motivation and career planning in QA. Alongside the development of QA staff, there is QA responsibility to train academic staff members on programme reviews, etc. A comprehensive, formal and structured approach is needed to address the matter of staff competence - a matter that should also be addressed across institutions. This could potentially be addressed as a national imperative.

A second focus area in this chapter is the integration of, and/or collaboration between, QA and IR. Although the majority of QA units are housed in the same bigger division (i.e. a division that includes other support units in addition to the QA unit), the integration of QA and IR, seems to be coincidental resulting in re-active integration. As far as QA's involvement in decision making is concerned, 50\% (seven) of the respondents indicated that they are not involved. Another three QA units indicated indirect or some involvement, depending on the issues at hand.

If an underpinning principle of QA, namely that it is developmental (i.e. improving quality), is taken into consideration, the collaboration/interaction between QA and IR becomes multidimensional. Both IR and QA should inform decision making, but their contributions differ; their approaches to data collection differ, the kinds of data/intelligence that they produce differ. QA staff are more visibly active in the academic domain and also in the service and support domain. They work closely with academic staff (not only with top or with middle management) and are involved in the development of the QA-related skills and knowledge of staff members across the institution. There should, first of all, be a good understanding and appreciation of the different kinds of contributions to institutional information. The best ways to collaborate and to integrate data to support the institution in achieving its strategic goals should be explored - again, not only institutionally, but also nationally.

\section{References}

Alfred, R.L. 2011. The future of institutional research. New Directions for Community Colleges, No 153.

Calderon, A. and Mathies, C. 2013. Institutional research in the future: Challenges within higher education and the need for excellence in professional practice. In: Calderon A \& Webber KL. (eds). Global Issues in Institutional Research. Number 157, 77-90.

Calderon, A. and Webber, K.L. (eds). Global Issues in Institutional Research. Number 157.

Council on Higher Education (CHE). 2004. Higher education quality committee.
Founding document. Second edition. Pretoria: $\mathrm{CHE}$

Council on Higher Education (CHE). 2014. Quality Enhancement Project. The process for public higher education institutions. Pretoria: Institutional Audits Directorate, 1-25.

Department of Education. 1997. Education White Paper 3. A programme for the Transformation of Higher Education. Pretoria: Department of Education.

Hannagan T. 1995. Management concepts and practices. Pitman Publishing: London. 
Jacobs, G.J., De Bruin, K. and Jacobs, M. 2013. Praktyke en strategieë wat ' $n$ kwaliteitsetos aan hoëronderwysinstellings behoort te koester. Tydskrif vir Geesteswetenskappe, 53:16-30.

Kinser K. 2014. Questioning quality assurance. New Directions for Higher Education, No 168, 55-68. http://dx.doi. org/10.1002/he.20113

Knight, W.E. and Leimer, C.L. 2010. Will IR staff stick? An exploration of institutional researchers' intention to remain in or leave their jobs. Research in Higher Education, Vol 51, 109-114. http:// dx.doi.org/10.1007/s11162-009-9152-9

Knight, W.E., Moore, M.E. and Copperthwaite, C.A. 1997. Institutional research: knowledge, skills and perceptions of effectiveness. Research in Higher Education, 38: 419-433. http:// dx.doi.org/10.1023/A:1024910409653

Knight, W.E. 2015. Developing the integrated institutional effectiveness office. Association for Higher Education Effectiveness: Webinar April 2015.

Lange L., Saavedra F.M. and Romano, J. 2013. Institutional research in emerging countries of Southern Africa, Latin America, and the Middle East and North Africa: Global frameworks and local practices. In: Calderon, A \& Webber, K.L. (eds). Global Issues in Institutional Research. Number 157. http://dx.doi. org/10.1002/ir.20037

Leimer, C. and Terkla, D.G. 2009. Laying the foundation: Institutional research office organization, staffing, and career development. New Directions for Institutional Research, No 143, 43-59. http://dx.doi.org/10.1002/ir.304

Leimer, C. 2012. Evidence-based decisionmaking and improvement. Change, July/ August 2012, 45-52. http://dx.doi.org/10 .1080/00091383.2012.691865

Materu, P.N. 2007. Higher Education Quality Assurance in Sub-Saharan Africa: Status, Challenges, Opportunities, and Promising Practices. Washington DC: The World Bank. http://dx.doi.org/10.1596/9780-8213-7272-2 PMid:17582900 PMCid:PMC2792864
Ministry of Education. May 2006. Ministerial statement on higher education funding: 2006/7 to 2008/9. Pretoria: Department of Education.

Schindler, L., Puls-Elvidge, S., Welzant, H., and Crawford, L. 2015. Definitions of quality in higher education: A synthesis of the literature. Higher Education Research Communications, 5(3), 3-13. http://dx.doi.org/10.18870/hlrc.v5i3.244

Sheldon, M.R., Golub A.J., Langevin J.R., St. Ours P.A. and Swartzlander B.J. 2008. Improving institutional effectiveness. Planning for Higher Education, JanuaryMarch, 17-26.

Strydom, A.H. 2001. Critical perspectives on quality assurance in higher education in South Africa. Commissioned paper in Transformation in higher education global pressures and local realities in South Africa. Bloemfontein: University of the Free State.

Taylor J., Hanlon, M. and Yorke, M. 2013. The evolution and practice of institutional research. In: Calderon A \& Webber KL. (eds). Global Issues in Institutional Research. Number 157, 5976. http://dx.doi.org/10.1002/ir.20039

Terenzini, P.T. 1993. On the nature of institutional research and the knowledge and skills it requires. Research in Higher Education, 34:1-10. http://dx.doi. org/10.1007/BF00991859

Terenzini, P.T. 2013. "On the Nature of Institutional Research" Revisited: Plus can Change...? Research in Higher Education, 54(2): 137-148. http://dx.doi. org/10.1007/s11162-012-9274-3

Webber, K.L. \& Calderon, A.J. 2015. Institutional research and planning in higher education: Global contexts and themes. New York \& London: Routledge Taylor and Francis Group.

Volkwein, J. 2008. The foundations and evolution of institutional research. New Directions for Higher Education, 141, 5-21. http://dx.doi.org/10.1002/he.289

White, E.K. 2007. Institutional effectiveness: The integration of program review, strategic planning, and budgeting processes in two California community colleges. DPhil dissertation. Andrews University: School of Education. 